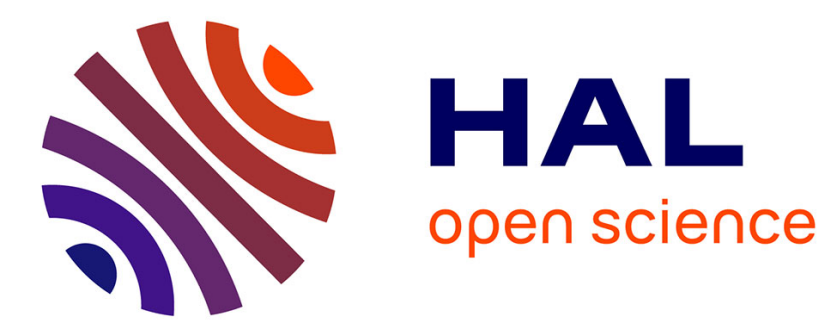

\title{
Organic field-effect transistor with octadecyltrichlorosilane (OTS) self-assembled monolayers on gate oxide: effect of OTS quality
}

\author{
M. Devynck, P. Tardy, G. Wantz, Y. Nicolas, L. Hirsch
}

\section{- To cite this version:}

M. Devynck, P. Tardy, G. Wantz, Y. Nicolas, L. Hirsch. Organic field-effect transistor with octadecyltrichlorosilane (OTS) self-assembled monolayers on gate oxide: effect of OTS quality. European Physical Journal: Applied Physics, 2011, 56 (3), pp.34106. 10.1051/epjap/2011110138 . hal-00756416

\author{
HAL Id: hal-00756416 \\ https://hal.science/hal-00756416
}

Submitted on 23 Nov 2012

HAL is a multi-disciplinary open access archive for the deposit and dissemination of scientific research documents, whether they are published or not. The documents may come from teaching and research institutions in France or abroad, or from public or private research centers.
L'archive ouverte pluridisciplinaire HAL, est destinée au dépôt et à la diffusion de documents scientifiques de niveau recherche, publiés ou non, émanant des établissements d'enseignement et de recherche français ou étrangers, des laboratoires publics ou privés. 


\title{
Organic Field-Effect Transistor with octadecyltrichlorosilane (OTS) Self-Assembled Monolayers on Gate oxide; effect of OTS quality
}

\author{
Authors: Mélanie Devynck ${ }^{(a)}$, Pascal Tardy ${ }^{(a)}$, Guillaume Wantz ${ }^{(a)}$, Yohann Nicolas ${ }^{(b)}$ and \\ Lionel Hirsch $^{(a)}$ \\ (a)Université de Bordeaux, Laboratoire d'Intégration du Matériau au Système (IMS), CNRS \\ UMR 5218 ENSCBP, 16 Av. Pey Berland 33607 Pessac Cedex, France \\ (b)Institut des Sciences Moléculaires (ISM), UMR 5255, Université Bordeaux, Bât. A12, 351 \\ Cours de la Libération, 33405 Talence Cedex, France
}

(e-mail adress : melanie.devynck@ims-bordeaux.fr)

\begin{abstract}
The effect of OTS (octadecyltrichlorosilane) Self Assembled Monolayer (SAM) grafted on $\mathrm{SiO}_{2}$ gate dielectric of pentacene based OFETs (organic field effect transistors) is investigated. A significant improvement of the charge mobility $(\mu)$, up to $0.74 \mathrm{~cm}^{2} / \mathrm{Vs}$, is reached thanks to OTS treatment. However, in spite of improved performances, several drawbacks, such as an increase in mobility dispersion, substantial hysteresis in $\mathrm{I}_{\mathrm{DS}}-\mathrm{V}_{\mathrm{G}}$ characteristics and high threshold voltages $\left(\mathrm{V}_{\mathrm{T}}\right)$ are observed. Changing solvent and deposition method turns out to have no significant effect on the mobility dispersion. A more accurate approach on the evolution of the mobility and the threshold voltage dispersion with OTS storage time highlights the effect of the OTS solution ageing. Even if no difference is evidenced in the surface energy and roughness of the OTS layer, electrical characteristics exhibit considerable deterioration with OTS solution storage time. Using an "aged" OTS solution, opened under air, kept under argon and distilled before use results in an increase of the $\mathrm{I}_{\mathrm{DS}}-\mathrm{V}_{\mathrm{G}}$ hysteresis as well as in $\mathrm{V}_{\mathrm{T}}$ and in mobility dispersion. In comparison, fresh-OTS-based OFETs present a very low hysteresis, a threshold voltage close to 0 and a much lower mobility dispersion. It is demonstrated that aged OTS solutions contain impurities that are not removed by distillation process which lead to less densely packed layer causing interfacial charge traps thus deteriorated performances.
\end{abstract}

\section{Introduction}

Within organic electronics, organic field effect transistors (OFETs) are of great interest for flexible displays, sensors and others applications where low cost fabrication is expected. Pentacene is one of the most promising organic semiconductor (OSC) due to its large field effect hole mobility (over $1 \mathrm{~cm}^{2} / \mathrm{Vs}$ ) [1-5]. However, the performances achieved vary greatly depending on the organic semiconductor / dielectric interface structure and thin film morphology. In OFETs, charge transport occurs within the first few monolayers of OSC close to its interface with the gate dielectric [6-8]. As a consequence electrical characteristics of OFETs are strongly related to the properties of the interfacial layers thus to the quality of the gate dielectric surface. Deteriorated performances are commonly associated with charge trapping at this interface due to a poor crystalline ordering inducing grain boundaries [9] or to dipolar interactions between the OSC and the polar $\mathrm{SiO}_{2}$ surface [10]. On the one hand, the OSC growth depends both on the roughness and the hydrophilicity of the dielectric. Pentacene grown on rougher substrates exhibits finer grain structure [11,12], hence higher density of grain boundaries acting as trap states. This point has been evidenced by Horowitz et al. on sexithiophene-based OFETs [13]. Several other studies have also pointed out a lowering of 
the mobility with an increasing roughness $[11,12]$. Besides, a polar (i.e. hydrophilic) dielectric such as $\mathrm{SiO}_{2}$ favors a three-dimensional Volmer-Weber OSC growth, increasing the number of in-plane boundaries and impeding charge transport [14]. On the other hand, the presence of interfacial-adsorbed water on the hydrophilic $\mathrm{SiO}_{2}$ surface can also be at the origin of randomly oriented dipoles acting as electronic trap states [15-18]. Self Assembled Monolayers (SAM) are widely used to modify the $\mathrm{SiO}_{2}$ dielectric surface. Due to dangling functional groups, the SAM can tune the dielectric surface energy [3], prevent adsorbtion of water molecules [19] and hence can reduce the charge traps density [20,21]. The first SAMsmodified gate dielectric was realized by Collet et al. in order to reduce the leakage current [22]. Further studies on pentacene OFETs revealed an increased mobility with the SAM dielectric modification. Hexamethyldisilasane (HMDS) [2], octadecyltrimethoxysilane (OTMS) [23] and octyltrichlorosilane (OTS) [19] on $\mathrm{SiO}_{2}$ allowed to reach mobilities of 3.4, 2.3 and $1.25 \mathrm{~cm}^{2} / \mathrm{Vs}$ respectively. Pentacene OFETs with alumina dielectric modified by alkyl group terminated phosphonic acid monolayers achieved mobilities of $2-3 \mathrm{~cm}^{2} / \mathrm{Vs}$ [24]. In this study, OTS is employed to modify the $\mathrm{SiO}_{2}$ gate dielectric. OTS is a convenient SAMs precursor since it bonds spontaneously on the $\mathrm{SiO}_{2}$ surface and does not require any post grafting treatment. In OSC, charge mobility is orders of magnitude lower than that in inorganic semiconductors, and OFETs are mostly considered for low frequency applications for which mobility is not a critical parameter. Contrarily, hysteresis which frequently appears on the $\mathrm{I}_{\mathrm{DS}}-\mathrm{V}_{\mathrm{G}}$ transfer curve and high threshold voltages are major concerns in most of these applications. In this purpose we mainly focus our study on the $\mathrm{I}_{\mathrm{DS}}-\mathrm{V}_{\mathrm{G}}$ curves hysteresis and threshold voltage. As expected, compared to raw $\mathrm{SiO}_{2}$ gate dielectric, OTS-modified OFETs, exhibit improved performances i.e. increased mobilities, absence of hysteresis and $V_{T}$ lowering. However, after several batches of OFETs, degradations appear such as discrepancies in mobility and $\mathrm{V}_{\mathrm{T}}$ and increased hysteresis, which are initially attributed to randomly ordered OTS monolayers generated by a deficient control of the SAM deposition stage. Influence of the grafting conditions investigated by varying solvents and deposition methods reveals that the dispersion and hysteresis keep increasing, even though control of the OTS monolayer quality by contact angle and AFM measurements reveal neither surface energy nor roughness alteration. Further investigations by Raman spectroscopy indicate that aged OTS solution contains certain impurities stemming from a high reactivity with air moisture. These impurities are not eliminated by the distillation stage and initiate defects in OTS monolayer explaining mobility and threshold voltage scattering as well as an increasing hysteresis of the $\mathrm{I}_{\mathrm{DS}}-\mathrm{V}_{\mathrm{G}}$ transfer curves. This work emphasizes the OTS solution quality as a key parameter in the reproducibility of OFETs performances.

\section{Experimental section}

OFETs devices were fabricated in a bottom contact geometry on commercial substrates (Fraunhofer IPMS). These substrates consisted in heavily doped Si wafer featuring a $230 \mathrm{~nm}$ $\mathrm{SiO}_{2}$ layer with patterned $30 \mathrm{~nm}$ Au electrodes on a $10 \mathrm{~nm}$ ITO adhesion layer. The electrodes set involved different channel lengths $(2.5,5,10$ and $20 \mu \mathrm{m})$ and a $1 \mathrm{~mm}$ width. Substrates were rinced with acetone, isopropanol followed by UV-ozone cleaning for $20 \mathrm{~min}$. The cleaning procedure of wafers was checked by contact angle measurements (drop of $3 \mu \mathrm{L}$ of ultrapure water) with a KRUSS DSA 100 goniometer. Before every SAMs grafting stage, commercially available OTS (Aldrich) was purified by distillation at $220^{\circ} \mathrm{C}$ under vacuum. In the case of the drop cast deposition, the OTS-based SAMs were formed by immersing the wafers in a $2.5 \mathrm{mM}$ cyclohexane:chloroform solution (70:30) (Aldrich, $\mathrm{H}_{2} \mathrm{O}<0.01 \%$ ) [25] or 
in a $2.5 \mathrm{mM}$ chlorobenzene solution (Aldrich, $\mathrm{H}_{2} \mathrm{O}<0.01 \%$ ) for 1 hour at $20^{\circ} \mathrm{C}$ under argon atmosphere. For spin coating deposition, the same solutions were employed and cast onto the substrate in order to entirely cover the surface and were allowed to partially assemble for 10s. Then, the substrate was spun at $3000 \mathrm{rpm}$ for $60 \mathrm{~s}$. After SAMs grafting, the wafers were rinsed with the deposition solvent and the grafting quality was checked by contact angle method with ultrapure water. Some samples were also prepared on glass substrate for InfraRed spectroscopy purpose following the same procedure. Pentacene was then vacuum evaporated $\left(1 \times 10^{-6} \mathrm{mbar}\right)$ at a rate of $0.1 \AA / \mathrm{s}$ to a total thickness of $40 \mathrm{~nm}$ in a temperaturecontrolled alumina-based crucible. The electronic characteristics of transistors were tested on a probe station using a KEITHLEY 4200 in a dry nitrogen glove box $\left(\mathrm{O}_{2}\right.$ and $\left.\mathrm{H}_{2} \mathrm{O}<1 \mathrm{ppm}\right)$. The mobilities were extracted from the saturation regime. Surface morphology of bare $\mathrm{SiO}_{2}$, OTS-Modified $\mathrm{SiO}_{2}$ and pentacene were probed by AFM (Veeco 3100, Digital Instrument) measurements. Raman spectra are performed on a LabRAM spectrometer (Horiba Jobin Yvon).

\section{Results and discussion}

The OTS monolayer deposition is initially performed by dropcasting the substrate in a (70:30) cyclohexane:chloroform solution $\mathrm{The}^{\mathrm{SiO}} \mathrm{O}_{2}$ based OFETs and the OTS-modified OFETs are electrically characterized and their performances: mobility $\mu$, threshold voltage $V_{T}$, hysteresis and $\mathrm{I}_{\mathrm{on}} / \mathrm{I}_{\text {off }}$ ratio are summarised in Table. 1. Mobilities are calculated in saturation regime and the hysteresis is defined as the difference between $V_{T}$ extracted from $I_{D S}-V_{G}$ curves for increasing and decreasing gate voltage $\mathrm{V}_{\mathrm{G}}$. The electrical parameters exposed in Table. 1 results from several independent batches of experiments. The normalised standard deviation NSD is calculated according to Eq.1.

$$
\mathrm{NSD}=\frac{\mathrm{SD}}{\overline{\mathrm{x}}}=\frac{\sqrt{\frac{1}{\mathrm{n}-1} \Sigma\left(\mathrm{x}_{\mathrm{i}}-\overline{\mathrm{x}}\right)^{2}}}{\overline{\mathrm{x}}} \quad \text { Eq. } 1
$$

Compared to bare $\mathrm{SiO}_{2}$, the modification of the dielectric by OTS improved the charge mobility by a factor 3 and allowed to reach a maximum mobility of $0.74 \mathrm{~cm}^{2} / \mathrm{Vs}$. Likewise, $V_{T}$, its associated NSD and $\mathrm{I}_{\mathrm{DS}}-\mathrm{V}_{\mathrm{G}}$ hysteresis decrease drastically while $\mathrm{I}_{\mathrm{on}} / \mathrm{I}_{\text {off }}$ exhibits no significant change. In spite of the increased mobility, a large discrepancy is observed on the OTS-based OFETs (see NSD in Table. 1). Random defects in the SAMs layer are believed to introduce this mobility dispersion. Experimental conditions have dramatic influence on the formation of the SAM. The characteristics (amount of water, dielectric constant) of the solvent employed to prepare the precursor OTS solution along with the grafting method [26] are all critical factors that affect the structure of the film. In the case of the alkyltrichlorosilane, the amount of water molecules in the solvent is a key parameter on the monolayer growth mechanism [27-29]. For anhydrous OTS solutions, the SAM film is formed in a two-step reaction, during the first stage, water molecules adsorbed on the hydrophilic $\mathrm{SiO}_{2}$ surface causes the hydrolysis of the OTS polar head group $\left(\mathrm{SiCl}_{3}\right)$ that converts $\mathrm{Si}-\mathrm{Cl}$ bonds to $\mathrm{Si}-\mathrm{OH}$ bonds releasing $\mathrm{HCl}$ molecules. Then, hydroxyl groups interact with each other and with the surface to form a covalent siloxane bonds (Si-O-Si) releasing one molecule of water for every bond formed. For water contaminated OTS solutions (water concentration $>50 \mathrm{mM}$ ) some OTS molecules appear to hydrolyse and react together to form large aggregates. These aggregates then react with the adsorbed water film or with the surface silanol groups and quickly cover the surface but are not able to form smooth 
monolayers as that observed with anhydrous solutions. Previous works have shown increased thickness and roughness of SAMs when OTS solutions were water-contaminated [30]. In order to prevent this drawback, OTS is distilled immediately before mixing with the solvent. Indeed, due to their higher molecular masses and supposed different boiling temperatures, aggregates and other impurities are supposedly eliminated by distillation. Besides, the dielectric constant of the solvent affects the coverage of the SAM molecules. Nie et al. have reported different behaviours depending whether SAMs have a polar head group and/or a non polar alkyl chain (as OTS) or not [31]. In the case of a solvent permittivity $\varepsilon_{\mathrm{s}}>5$, the SAM molecules start to interact significantly with the solvent, thus disrupting the self-assembly on the substrate. However, if $\varepsilon_{\mathrm{s}}<3$, SAM molecules tend to make reverse micelles in solution which give rise to incomplete coverage of the SAM layer. Hence, the amount of water and the dielectric permittivity of the solvent are crucial parameters for SAM growth. In addition, in previous work, Bao's group shown that the molecular ordering of the SAM layer depends also on the deposition method [23,26]. As a poor control of the SAMs grafting stage is suspected to be responsible for $\mu$ and $\mathrm{V}_{\mathrm{T}}$ scattering just as for hysteresis, alternative deposition stages are experienced changing the solvent and/or grafting method. In a same batch, distilled OTS molecules are grafted, by dropcasting or by spincoating with two different solvents (cyclohexane:chloroform or chlorobenzene). After grafting, the OTS monolayer is characterized by IR spectroscopy and by AFM. and its surface energy monitored by ultrapure water contact angle. The IR spectroscopy (Fig. 1) shows no significant difference in the position of antisymmetric $\left(2917 \mathrm{~cm}^{-1}\right)$ and symmetric $\left(2850 \mathrm{~cm}^{-1}\right) \mathrm{CH}_{2}$ stretching peaks revealing the SAM ordering [32]. However, as it can be observed in Table. 2, OTS layer roughness clearly depends on the type of solvent and on the deposition method, the best results (i.e. lowest OTS roughness) being obtained with drop casting in cyclohexane:chloroform solution (protocol 1). After pentacene deposition, OFETs are electrically characterized and key parameters: mobility $\mu$, threshold voltage $V_{T}$ and $I_{o n} / I_{o f f}$ ratio are presented in Table. 2 . In coherence with roughness results, the highest mobilities and $\mathrm{I}_{\mathrm{on}} / \mathrm{I}_{\mathrm{off}}$ as well as the lowest $\mathrm{V}_{\mathrm{T}}$ are achieved with protocol 1 . In addition, a better reproducibility of the mobility, i.e. a lower NSD and a less pronounced hysteresis are achieved. Consequently, although changing the solvent nature and the deposition method were not found to reduce the dispersion previously observed (Table. 1), these results validate our initial choice for SAM grafting procedure.

Nevertheless, OFETs fabricated according to this procedure exhibit deteriorated performances compared to that initially obtained in the same conditions, which drive us to assume that OTS solution ageing could be at the origin of the degradation, independently of the grafting protocol. Therefore, in order to verify this assumption, several batches of OFETs are fabricated at different times with grafted-OTS monolayer, according to protocol 1 with the same bottle of OTS and electrically characterized. Between each utilisation, the OTS bottle is sealed under argon atmosphere and shelved. Before mixing with the solvent, OTS is purified by distillation. Indeed, the $\mathrm{Si}-\mathrm{Cl}$ bonds of the OTS head group can easily react with water to form aggregates and/or Si-OH bonds resulting in a poor SAM layer quality as mentioned above. After grafting, the surface energy of OTS monolayer is monitored by ultrapure water contact angle and its roughness determined by AFM measurements (Fig. 2). The results presented versus time after OTS bottle aperture in Fig. 3 show no significant alteration of the contact angle which remains close to values measured in other works [33] idem for roughness (rms $\sim 0.2 \mathrm{~nm}$ ). Eventually, OFETs were fabricated from the OTS-modified substrates and electrically characterized. Mobilities and threshold voltage are presented in Fig. 4(A) and 4(B) respectively, if some variations are noticeable, no general relation with OTS ageing can be posited so far. Conversely, discrepancy on $\mu$, and $V_{T}$ as long as $\mathrm{I}_{\mathrm{DS}}-\mathrm{V}_{\mathrm{G}}$ transfert curves 
hysteresis (Fig.5) increased dramatically, indicating serious device deterioration. As it can be noticed in Fig. 6, a sample prepared from a freshly opened OTS bottle $\left(\mathrm{t}_{0}\right)$ and electrically tested exhibits no hysteresis and a value of $\mathrm{V}_{\mathrm{T}}$ closer to 0 , confirming the deleterious influence of OTS ageing. Hysteresis is commonly ascribed to charge trapping at the dielectric-semiconductor interface [9,34,35], hydroxyl groups at the dielectric surface being supposed to generate electronic traps. At this stage, since the morphological aspects cannot be responsible for the electrical performances difference between the samples just as it has been highlighted in former works [23], the purification process is suspected to be inefficient. In case of densely packed OTS layers achieved by the use of pure OTS solution, $\mathrm{Si}_{-} \mathrm{Cl}_{3}$ head groups react with the water molecules present at the $\mathrm{SiO}_{2}$ surface. This case corresponds to the grafting mechanism described above in case of anhydrous OTS solutions. However, in an aged OTS solution (which can be compared to the growth mechanism in case of water concentration $>50 \mathrm{mM}$ ), Si-OH bonds remains despite the distillation. These bonds present before the grafting induce aggregates preventing an effective grafting of the OTS monolayer. It results a poor $\mathrm{SiO}_{2}$ surface coverage and occurrence of remaining hydroxyl groups inducing electronic charge traps at the interface. In order to check this assumption Raman spectra of two OTS solutions samples are performed: both of them stem from the same OTS bottle opened several times under air and kept under argon for 2 months but one sample is distilled immediately before characterization while the second sample is not. The Raman spectroscopy (Fig. 7) evidences a Si-OH peak $\left(830-920 \mathrm{~cm}^{-1}\right)$ [36] in both samples spectra indicating that distillation is not a convenient purification process for OTS solution and confirms that OTS ageing is detrimental for OFETs electrical hysteresis.

\section{Conclusion}

In summary, it has been shown that the $\mathrm{SiO}_{2}$ modification with OTS yield to charge mobility increase as well as threshold voltage and $\mathrm{I}_{\mathrm{DS}}-\mathrm{V}_{\mathrm{G}}$ transfer curves hysteresis decrease. The best results are obtained by drop casting in cyclohexane:chloroform (70:30) OTS solution. The OTS-grafted OFETs exhibit increasing discrepancies in mobilities and threshold voltages along with dramatically amplified hysteresis as a function of OTS solution storage time although no significant difference in surface energy or in roughness are measured. Average mobility and threshold voltage appear to be also noticeably affected by OTS ageing but with no significant trend. It is found that the OTS solution get partially hydrolysed with storage time, which results in impurities that cannot be removed with distillation leading to a less densely packed layer and altered electrical performances. This study has emphasized the importance of the OTS solution quality in the SAM growth. To reach high reproducibility, the use of fresh OTS solution never exposed to air is recommended.

The authors would like to thank G. Le Bourdon (ISM laboratory) for the Raman spectroscopy support and C. Labrugere (ICMCB laboratory) for the AFM microscopy support. 


\begin{tabular}{|l|c|c|c|c|}
\hline Dielectric surface & Average $\mu\left[\mathrm{cm}^{2} / \mathrm{Vs}\right.$ ] (NSD) & $\mathrm{V}_{\mathrm{T}}[\mathrm{V}](\mathrm{NSD})$ & $\mathrm{I}_{\text {on }} / \mathrm{I}_{\text {off }}$ & Average hysteresis [V] \\
\hline Bare $\mathrm{SiO}_{2}$ & $0.1(1)$ & $-4(4.5)$ & $1.3 \times 10^{7}$ & 11.35 \\
\hline OTS & $0.20(0.9)$ & $-2(0.2)$ & $1.2 \times 10^{7}$ & 0.75 \\
\hline
\end{tabular}

Table. 1. Summary of the electrical results depending on the dielectric surface: $\mathrm{SiO}_{2}$ or OTS-treated $\mathrm{SiO}_{2}$. The values shown are averages over $\sim 120$ devices for each dielectric surface treatment. 


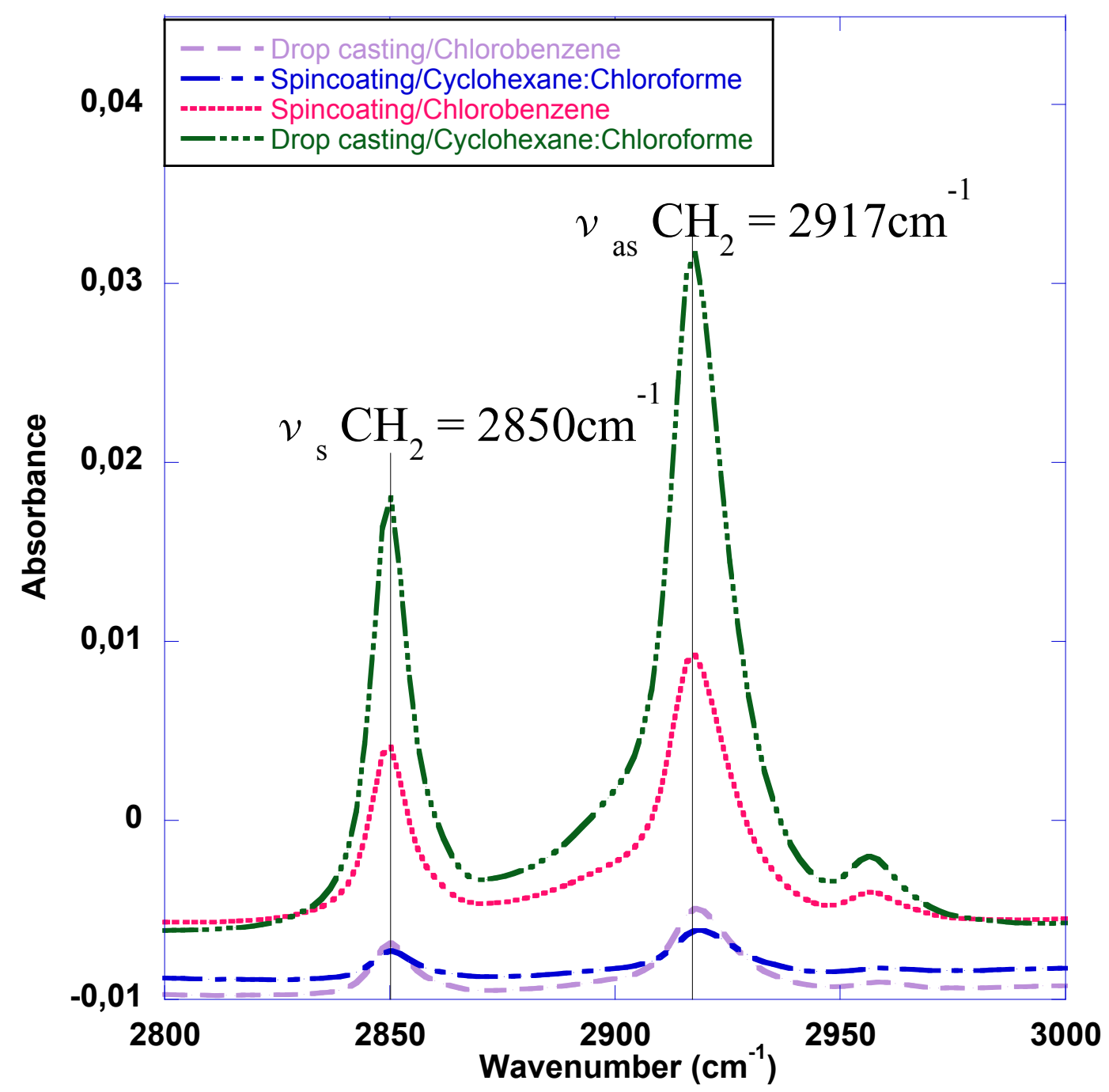

Fig. 1. IR spectra of the OTS layer deposited on glass substrates depending on the deposition method. 


\begin{tabular}{|c|c|c|c|c|c|c|c|}
\hline $\begin{array}{c}\text { Deposition } \\
\text { method }\end{array}$ & Solvent & $\begin{array}{c}\text { Average } \mu \\
{\left[\mathrm{cm}^{2} / \mathrm{Vs}\right](\mathrm{NSD})}\end{array}$ & $\begin{array}{c}\text { Average } \\
\mathrm{V}_{\mathrm{T}}[\mathrm{V}]\end{array}$ & $\begin{array}{c}\text { Average } \\
\mathrm{I}_{\mathrm{on}} / \mathrm{I}_{\mathrm{off}}\end{array}$ & $\begin{array}{c}\text { Average } \\
\text { hysteresis } \\
{[\mathrm{V}]}\end{array}$ & $\begin{array}{c}\text { Rms } \\
\text { roughness } \\
{[\mathrm{nm}]}\end{array}$ & $\begin{array}{c}\text { Average } \\
\text { contact } \\
\text { angle } \\
{\left[{ }^{\circ}\right]}\end{array}$ \\
\hline $\begin{array}{c}\text { Drop } \\
\text { casting }\end{array}$ & Cyclohexane:chloroform & $0.16(0.44)$ & 0.8 & $6.5 \times 10^{5}$ & 0.3 & 0.4 & 106.6 \\
\hline $\begin{array}{c}\text { Drop } \\
\text { casting }\end{array}$ & Chlorobenzene & $0.02(0.68)$ & 2.8 & $8.4 \times 10^{4}$ & 0.88 & 1 & 108.3 \\
\hline $\begin{array}{c}\text { Spin } \\
\text { casting }\end{array}$ & Cyclohexane:chloroform & $0.040 .65)$ & 1.5 & $2.9 \times 10^{4}$ & 3.4 & 1.3 & 106 \\
\hline $\begin{array}{c}\text { Spin } \\
\text { casting }\end{array}$ & Chlorobenzene & $0.07(0.57)$ & 3.7 & $2.5 \times 10^{4}$ & 2.05 & 6 & 106 \\
\hline
\end{tabular}

Table. 2. Electrical performances and OTS layer roughness and contact angle for different deposition conditions. Each experiment results in the average of 16 devices. 


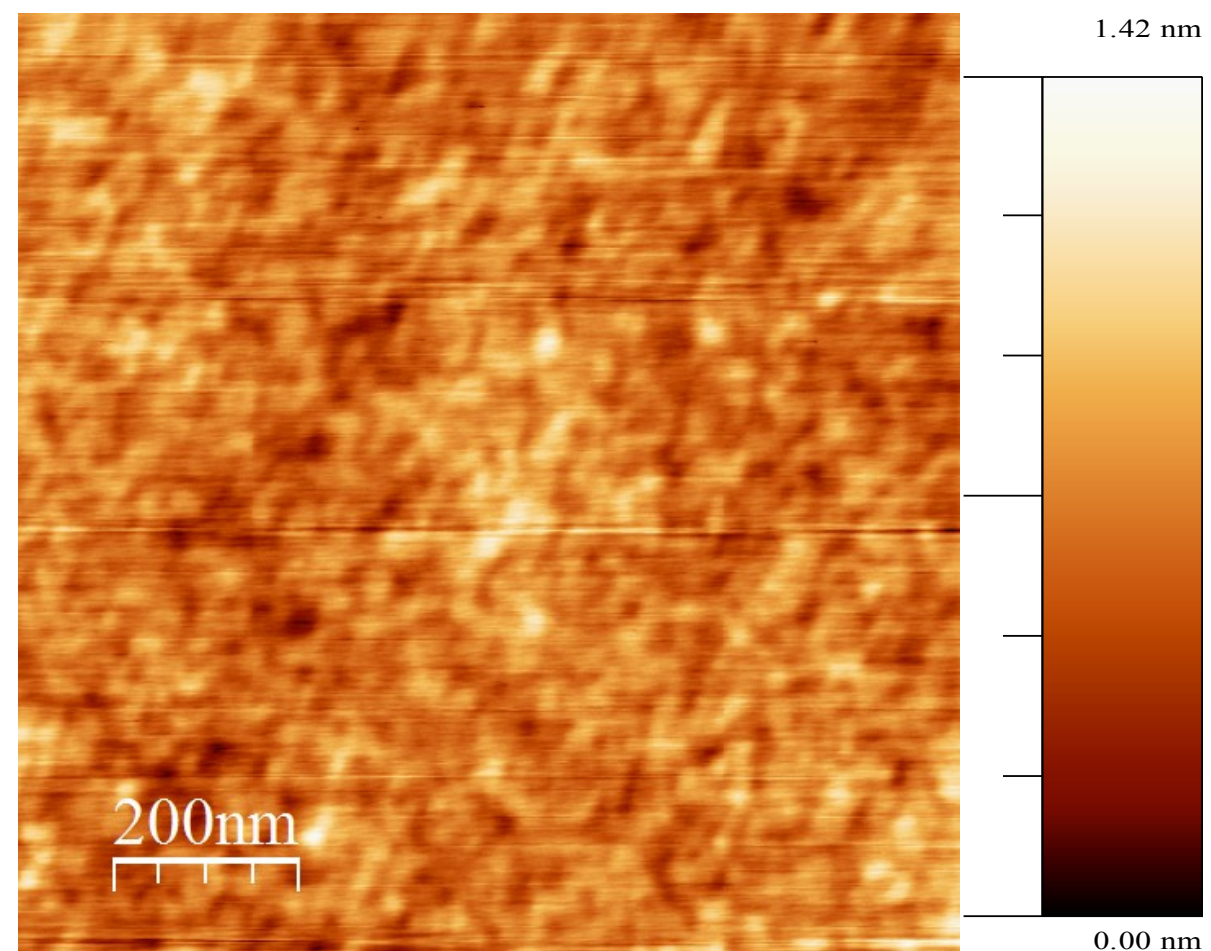

Fig. 2. Topographic AFM image $(1 \mu \mathrm{m} \times 1 \mu \mathrm{m})$ of an OTS layer grafted with the protocol 1 . 


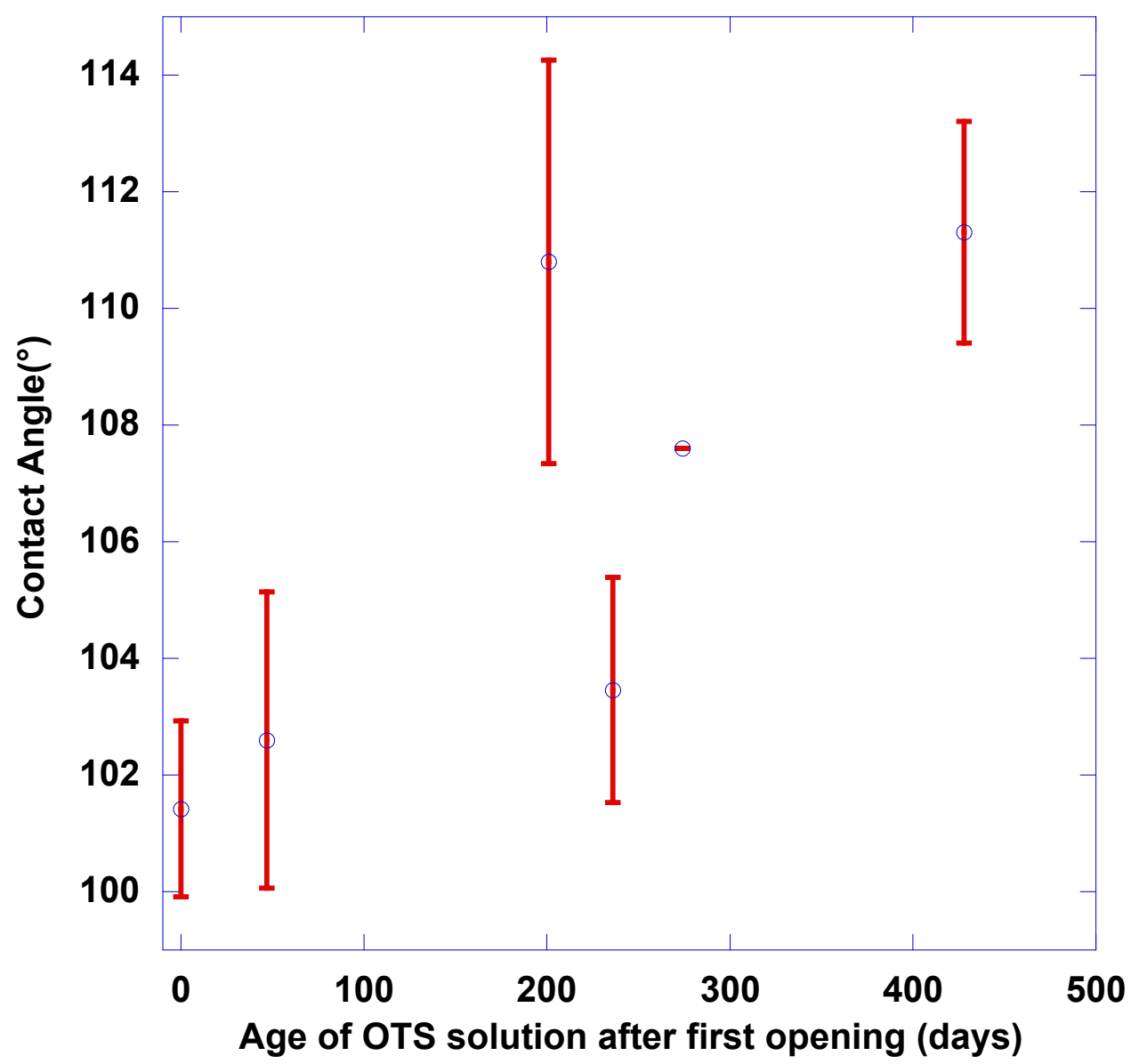

Fig. 3. Evolution of the contact angle with the OTS solution ageing 

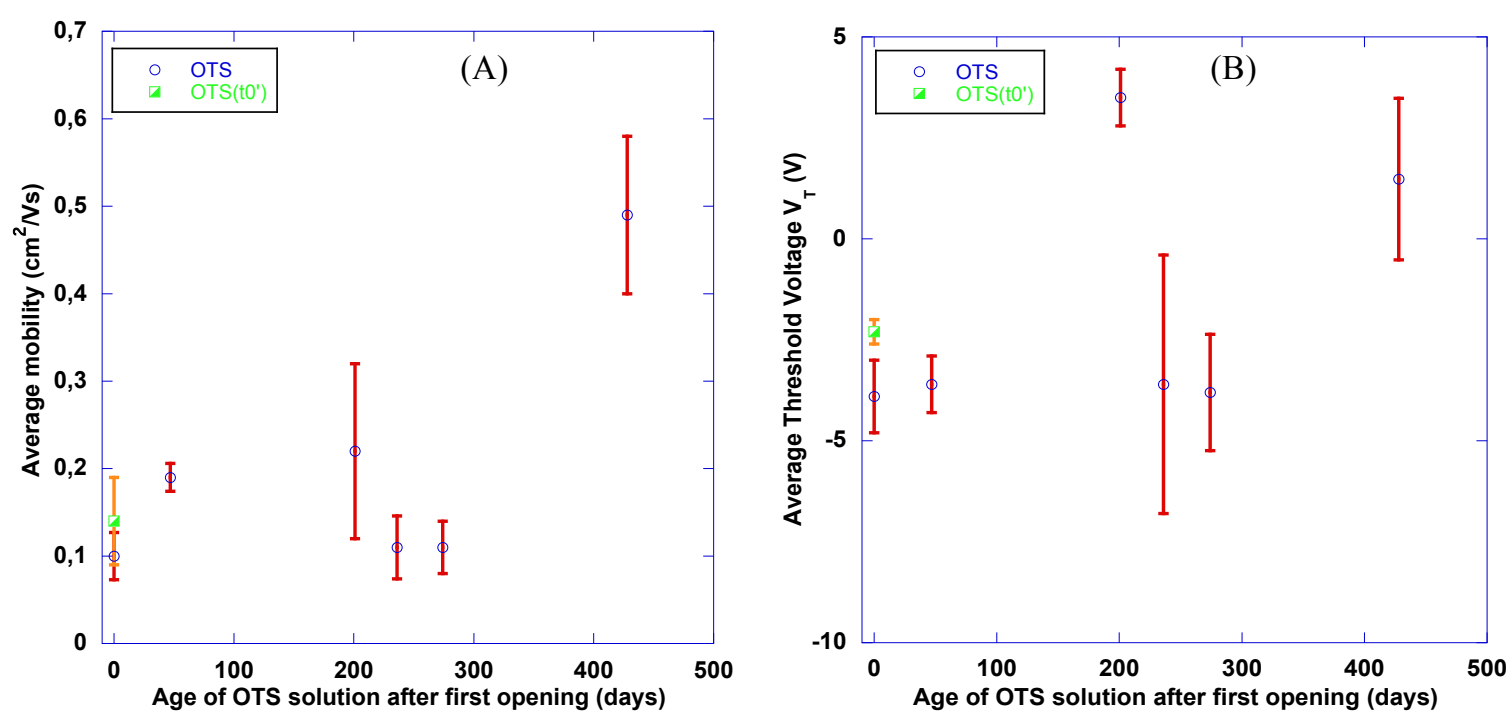

Fig. 4. Evolution of (A) the average mobility $\mu$ and of (B) the threshold voltage $\mathrm{V}_{\mathrm{T}}$ with the OTS solution ageing. The value at $\mathrm{t}_{0}$ ' refers to the control sample prepared from a newly opened OTS bottle $\left(\mathrm{t}_{0}\right.$ '), i.e. in the same conditions as at $\mathrm{t}=0$ days. 


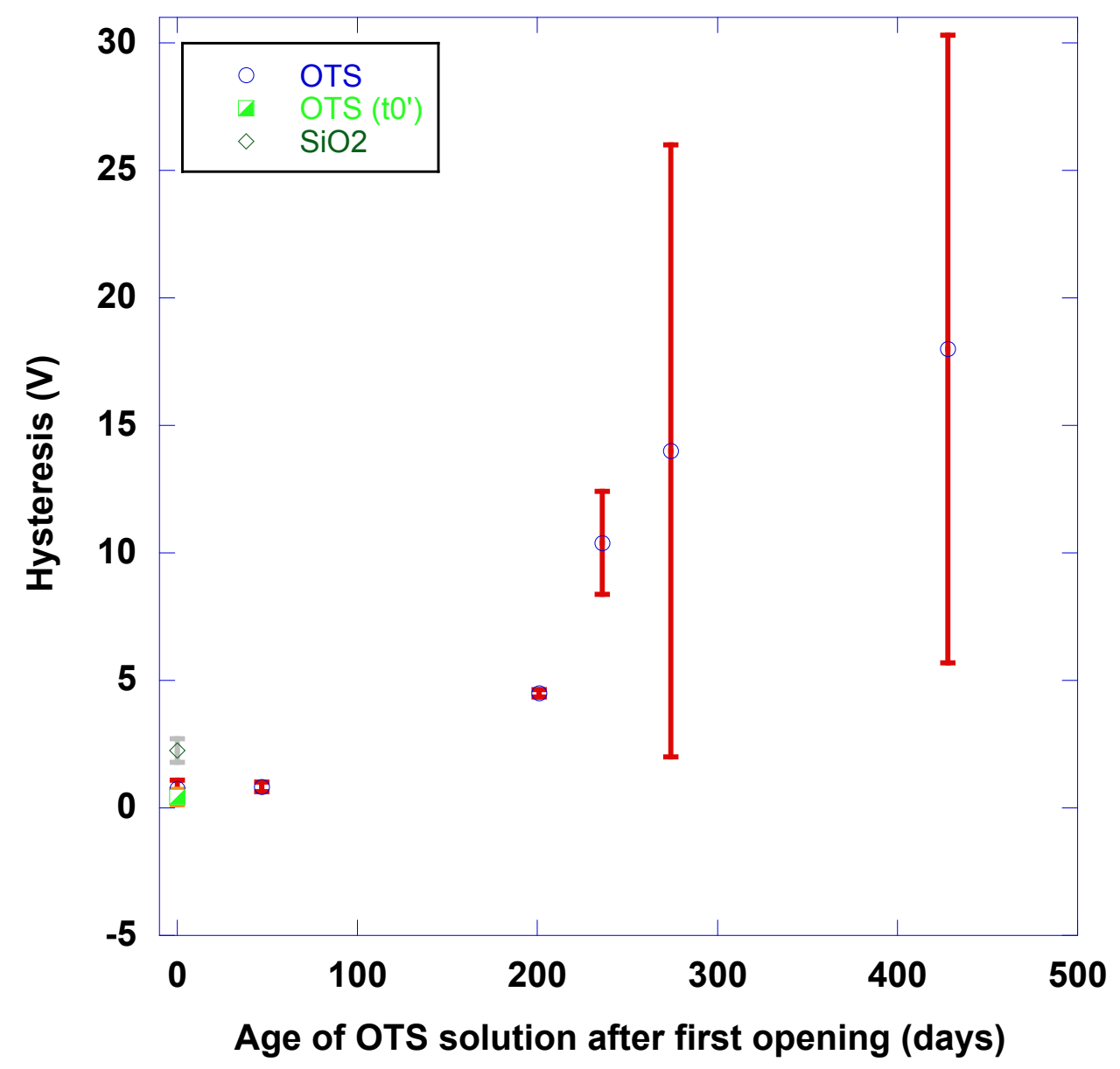

Fig. 5. Evolution of the $\mathrm{I}-\mathrm{V}$ hysteresis with the OTS solution ageing. The $\mathrm{SiO}_{2}$ hysteresis is added to comparison. The value at $\mathrm{t}_{0}$ ' refers to the control sample prepared from a newly opened OTS bottle $\left(\mathrm{t}_{0}{ }^{\prime}\right)$, i.e. in the same conditions as at $\mathrm{t}=0$ days. 

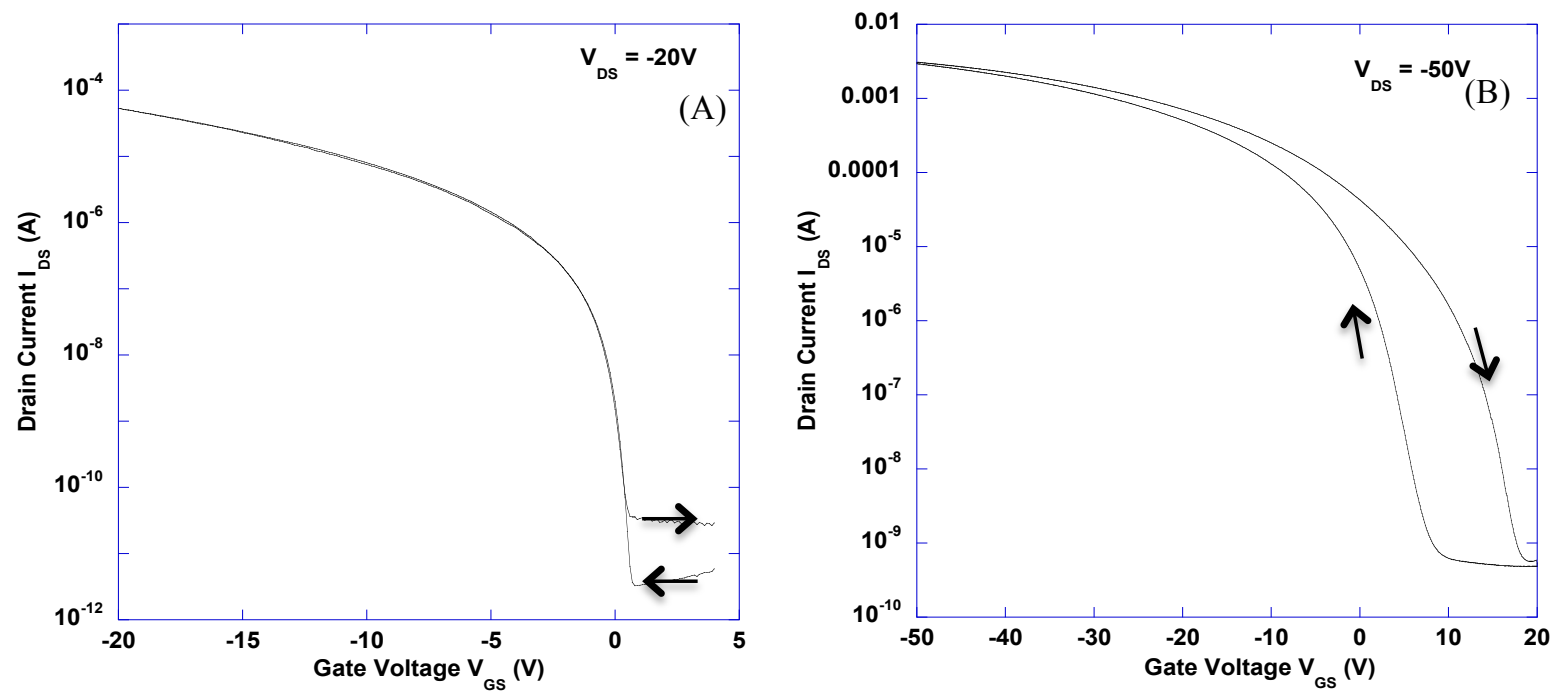

Fig. 6. Transfert characteristics of a transistor prepared at $t_{0}(A)$ and at $t=201$ days $(B)$. 


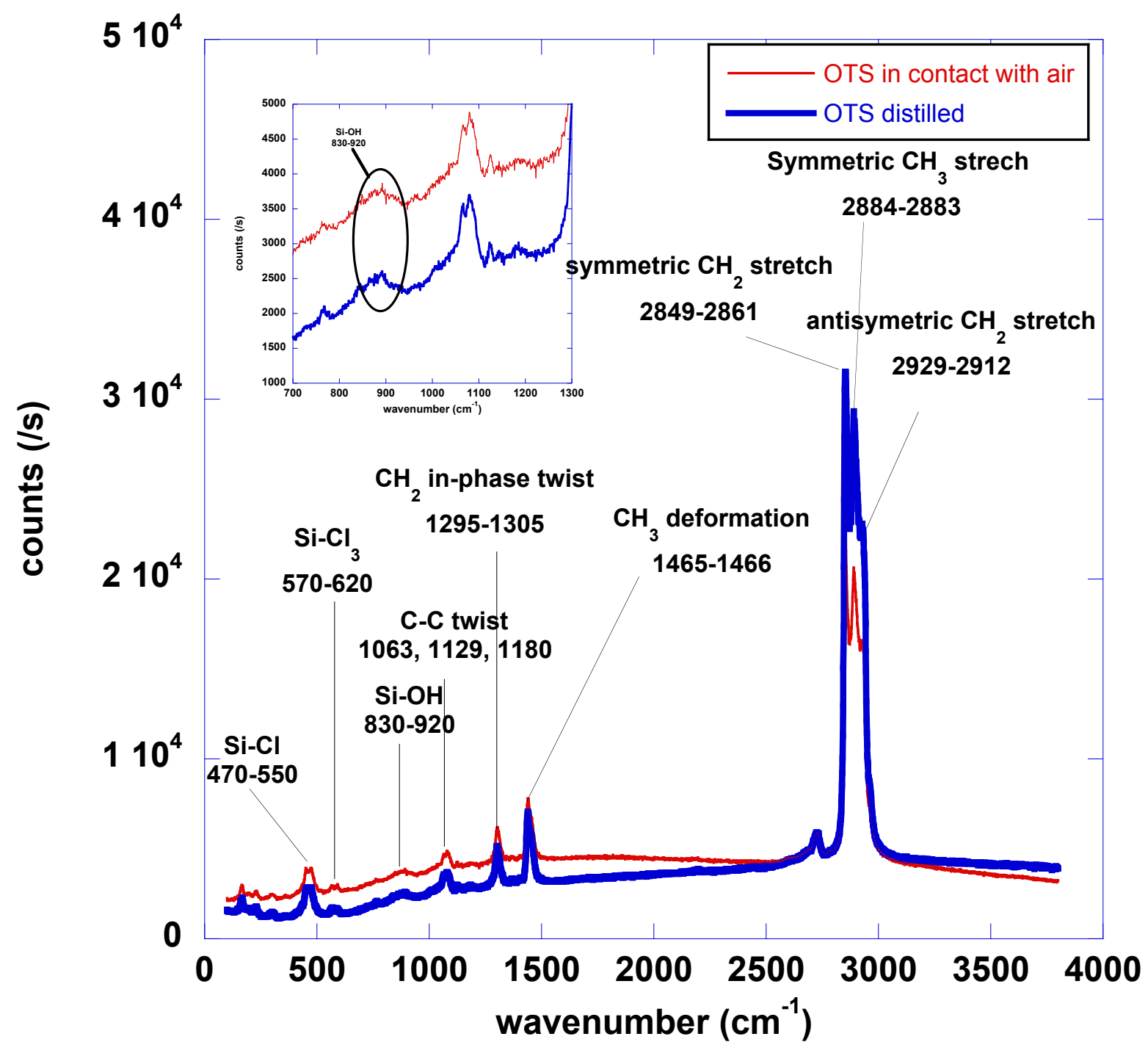

Fig. 7. Raman spectra of the two OTS solutions: the OTS solution opened several times under argon and kept under argon and the OTS solution distilled immediately before the characterization. The inset is a zoom in of the $700-1300 \mathrm{~cm}^{-1}$ range highlighting Si-OH peaks from impurities. 
1. C. D. Sheraw, L. Zhou, J. R. Huang, D. J. Gundlach, T. N. Jackson, M. G. Kane, I. G. Hill, M. S. Hammond, J. Campi, B. K. Greening, J. Francl, and J. West, Appl. Phys. Lett. 80, 1088 (2002).

2. H. Yang, T. J. Shin, M-M Ling, K. Cho, C. Y. Ryu, and Z. Bao, J. Am. Chem. Soc. 127, 11542-11543 (2005).

3. K. Shankar and T. N. Jackson, J. Mat. Res. 19, 2003-2007 (2004).

4. Y. Kato, S. Iba, R. Teramoto, T. Sekitani, T. Someya, H. Kawaguchi, and T. Sakurai, Appl. Phys. Lett. 84, 3789 (2004).

5. D. J. Gundlach, T. N. Jackson, D. G. Schlom, and S. F. Nelson, Appl. Phys. Lett. 74, 3302 (1999).

6. M. Kiguchi, M. Nakayama, T. Shimada, and K. Saiki, Phys. Rev. B 71, 1-5 (2005).

7. F. Dinelli, M. Murgia, P. Levy, M. Cavallini, F. Biscarini, and D. De Leeuw, Phys. Rev. Lett. 92, 90-93 (2004).

8. G Horowitz, P Lang, W Kalb, and M Mottaghi, in Proceedings of the International Symposium in Super-Functionality Organic Devices, IPAP Conf. Series 6, 2005, p.125-129.

9. G. Horowitz, M.E. Hajlaoui, and R. Hajlaoui, J. Appl. Phys. 87, 4456 (2000).

10. J. Veres, S.D. Ogier, S.W. Leeming, D.C. Cupertino, and S. Mohialdin Khaffaf, Adv. Funct. Mater. 13, 199-204 (2003).

11. S. Steudel, S. De Vusser, S. De Jonge, D. Janssen, S. Verlaak, J. Genoe, and P. Heremans, Appl. Phys. Lett. 85, 4400 (2004).

12. S. E. Fritz, T. W. Kelley, and C. D. Frisbie, J. Phys. Chem. B 109, 10574-7 (2005).

13. G Horowitz and M.E. Hajlaoui, Synth. Met. 122, 185-189 (2001).

14. G. Yoshikawa, J. T. Sadowski, A. Al-Mahboob, Y. Fujikawa, T. Sakurai, Y. Tsuruma, S. Ikeda, and K. Saiki, Appl. Phys. Lett. 90, 251906 (2007).

15. S Wo, B Wang, H Zhou, Y Wang, J Bessette, R. L Headrick, A C Mayer, G G Malliaras, and A Kazimirov, J. Appl. Phys. 100, 093504 (2006).

16. A Kazimirov and G G Malliaras, with A C Mayer and R Ruiz, Headrick R.L, Org. Elec. 5, 257-263 (2004).

17. R Ruiz, A Papadimitratos, A C Mayer, and G G Malliaras, Adv. Mater. 17, 1795 (2005).

18. R. Ruiz, B. Nickel, N. Koch, L. Feldman, R. Haglund, A. Kahn, and G. Scoles, Phys. Rev. B 67, 1-7 (2003). 
19. H Seo, Y Jang, Y Zhang, P Syedabthagir, and J Choi, Org. Elec. 9, 432-438 (2008).

20. C. Goldmann, D. J. Gundlach, and B. Batlogg, Appl. Phys. Lett. 88, 063501 (2006).

21. H Ohnuki, W Changhai, M Izumi, Y Tatewaki, and K Ikegami, Thin Solid Films 516, 2747-2752 (2008).

22. J Collet and D Vuillaume, Appl. Phys. Lett. 73, 2681 (1998).

23. A. Virkar, S. Mannsfeld, J.H. Oh, M.F. Toney, Y.H. Tan, G. Liu, J.C. Scott, R. Miller, and Z. Bao, Adv. Funct. Mater. 19, 1962-1970 (2009).

24. T. W. Kelley, L. D. Boardman, T. D. Dunbar, D. V. Muyres, M. J. Pellerite, and T. P. Smith, J. Phys. Chem. B 107, 5877-5881 (2003).

25. A N Parikh, D L Allara, I B Azouz, and F Rondelez, J. Phys. Chem. 98, 7577-7590 (1994).

26. Y. Ito, A. A. Virkar, S. Mannsfeld, J. H. Oh, M. Toney, J. Locklin, and Z. Bao, J. Am. Chem. Soc. 131, 9396-9404 (2009).

27. L.-J. Chen, Y-H. Tsai, C.-S. Liu, D.-R. Chiou, and M.-C. Yeh, Chem. Phys. Lett. 346, 241-245 (2001).

28. S. Pillai and R. K. Pai, Ultramicroscopy 109, 161-6 (2009).

29. B. C. Bunker, R. W. Carpick, R. A. Assink, M. L. Thomas, M. G. Hankins, J. A. Voigt, D. Sipola, M. P. de Boer, and G. L. Gulley, Langmuir 16, $7742-7751$ (2000).

30. Y. Wang and M. Lieberman, Langmuir 19, 1159-1167 (2003).

31. H-Y Nie, M. J. Walzak, and N. S. McIntyre, J. Phys. Chem. B 110, 21101-8 (2006).

32. H. S. Lee, D. H. Kim, J. H. Cho, M. Hwang, Y. Jang, and K. Cho, J. Am. Chem. Soc. 130, 10556-64 (2008).

33. D K Aswal, S Lenfant, D Guerin, J V Yakhmi, and D Vuillaume, Analytica Chimica Acta 568, 84-108 (2006).

34. G. Gu, M. G. Kane, J. E. Doty, and A. H. Firester, Appl. Phys. Lett. 87, 243512 (2005).

35. H. E. Katz, X. M. Hong, A. Dodabalapur, and R. Sarpeshkar, J. Appl. Phys. 91, 1572 (2002).

36. D Lin-Vien, W N Colthup, W G Fateley, and J G Grasselli, The Handbook Of Infrared and Raman Frequencies Of Organic Molecules (John Wiley \& Sons, Ltd., 1991). 
